\title{
Heterogeneous excitable systems exhibit Griffiths phases below hybrid phase transitions
}

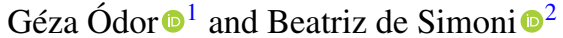 \\ ${ }^{1}$ Institute of Technical Physics and Materials Science, Center for Energy Research, P.O. Box 49, H-1525 Budapest, Hungary \\ ${ }^{2}$ Budapest University of Technology and Economics, Müegyetem rkp. 3., H-1111 Budapest, Hungary
}

(Received 2 December 2020; accepted 21 January 2021; published 2 February 2021)

\begin{abstract}
In $d>2$ dimensional, homogeneous threshold models discontinuous transition occur, but the mean-field solution provides $1 / t$ power-law activity decay and other power laws, and thus it is called mixed-order or hybrid type. It has recently been shown that the introduction of quenched disorder rounds the discontinuity and second-order phase transition and Griffiths phases appear. Here we provide numerical evidence that even in case of high graph dimensional hierarchical modular networks a Griffiths phase in the $K=2$ threshold model is present below the hybrid phase transition. This is due to the fragmentation of the activity propagation by modules, which are connected via single links. This provides a widespread mechanism in the case of the threshold type of heterogeneous systems, modeling the brain, or epidemics for the occurrence of dynamical criticality in extended Griffiths phase parameter spaces. We investigate this in synthetic modular networks with and without inhibitory links as well as in the presence of refractory states.
\end{abstract}

DOI: 10.1103/PhysRevResearch.3.013106

\section{INTRODUCTION}

Phase transitions in genuine nonequilibrium systems have often been investigated among the reaction-diffusion (RD) type of models exhibiting absorbing states [1,2]. In many cases mapping to surface growth, spin systems, or stochastic cellular automata has been used. Criticality allows us to define universality classes, defined by the scaling exponents, which have been explored in homogeneous systems $[3,4]$. In heterogeneous network models the situation is less clear. Hybrid phase transition (HPT) means that at the transition point the order parameter exhibits a jump, in conjunction with critical phenomena related to it. It can mean avalanches of activity at the transition point with power-law (PL) size distribution, for example. Such types of transitions have been known for a long time [5], for example, at tricriticality [6,7], but had not been the focus of research and the term appeared later. HPTs have been found in network science in the case of $k$-cores [8], interdependent networks [9], and multiplexes [10].

The "mixed-order" name for the same phenomena in statistical physics arose some years ago [11] by the exactly soluble one-dimensional Ising model with long-range interactions. It is also known to appear in nonequilibrium models, exhibiting a transition to absorbing states [12]. Further examples include critical models at extended surface defects $[13,14]$ and synchronization [15-17].

Criticality is a ubiquitous phenomenon in nature as systems can benefit in many ways from it. As correlations and fluctu-

Published by the American Physical Society under the terms of the Creative Commons Attribution 4.0 International license. Further distribution of this work must maintain attribution to the author(s) and the published article's title, journal citation, and DOI. ations diverge [18] in neural systems, working memory and long connections can be generated spontaneously [19] and the sensitivity to external signals is maximal. Furthermore, it has also been shown that information-processing capabilities are optimal near the critical point. Therefore, systems tune themselves close to criticality via self-organization (SOC) [20,21], presumably slightly below to avoid blowing overexcitation. Besides, if quenched heterogeneity (which is called disorder compared to a homogeneous system) is present, rare-region effects (RRs) [22] and an extended semicritical region, known as a Griffiths phase (GP) [23], can emerge. RRs are very slowly relaxing domains, remaining in the opposite phase than the whole system for a long time, causing slow evolution of the order parameter. In the entire GP, which is an extended control parameter region around the critical point, susceptibility diverges and autocorrelations exhibit fat-tailed, power-law behavior, resulting in bursty behavior [24], frequently observed in nature [25]. Even in infinite dimensional systems, where mean-field behavior is expected, Griffiths effects [26] can occur in finite time windows.

It is known that strong disorder can round or smear phase transitions [22]. According to the arguments by Imry-Ma [27] and Aizenman-Wehr [28], first-order transitions do not exist in low-dimensional disordered systems. It has recently been shown $[29,30]$ that this is true in genuinely nonequilibrium systems $[1,4]$.

Experimental and theoretical research provide evidence that the brain operates in a critical state between sustained activity and an inactive phase [18,31-36]. Criticality in general occurs at continuous, second-order phase transitions. On the other hand, metastability and hysteresis are also common in the brain behavior. They are related to the ability to sustain stimulus-selective persistent activity for working memory [37]. The brain rapidly switches from one state to another in response to stimulus, and it may remain in the 
same state for a long time after the end of the stimulus. It suggests the existence of a repertoire of metastable states. Several models have described this [38,39]. It introduces an apparent contradiction, because metastability and hysteresis occur in general at first-order, discontinuous phase transitions. But the brain can operate at different regimes close to the critical point, which can provide the desired advantages for biological systems. Another possible resolution for the above controversy is the operation at a transition of hybrid type. It has also been suggested in a recent theoretical work [40].

Threshold types of systems, like the integrate-and-fire models of the brain [41], are also suggested to describe other phenomena, like power grids [42-44], crack and fracture formation [45], contagion [46], etc. In these models HPT can emerge naturally, and thus the present results can also be relevant.

Heterogeneity effects are very common in nature and result in dynamical criticality in extended GPs, in the case of quasistatic quenched disorder approximation [47]. This leads to avalanche size and time distributions, with nonuniversal PL tails. It has been shown within the framework of modular networks [47-49] and a large human connectome graph [50]. In this study we reuse the hierarchical modular network of Ref. [48] and provide numerical evidence that above the GP a HPT emerges. Metastable states and hysteresis behavior can also be found; thus this system can oscillate between up and down states, depending on the level of oscillations, without the need of oscillators at the nodes, as in the case of the Ginzburg-Landau theory, suggested to model cortical dynamics [51]. By extending our model we will show that the proposed mechanism is very general, providing an explanation for the observed wide range of scale-free behavior below the transition point.

\section{MEAN-FIELD APPROXIMATION}

Discrete threshold models can be defined as two-state systems: $x_{i}=0,1$ (inactive, active) at sites $i$, with a conditional activation rule, depending on the sum of activity of neighbors compared to the threshold value $K$ :

$$
\sum_{j} x_{j} w_{i, j} \geqslant K,
$$

where $w_{i, j}$ is the weight of the link connecting site $j$ to $i$. In interacting homogeneous systems $w_{i, j}$ is just the adjacency matrix element, $A_{i, j}$, which is 1 if nodes are connected or 0 otherwise. To describe stochasticity this activity creation can be accepted with probability $\lambda$, competing with an activity removal process of probability $\nu$. The mean-field description of the threshold model of $N$ nodes can be obtained in a similar way as in the case of RD systems [52]. That work is defined on the lattice, but we can apply it for other graphs. In Ref. [52] it was shown that a discontinuous jump occurs in mean-field models of the $n>m$ RD systems, in which $n$ neighboring particles are needed for creation and $m$ neighbors for removal. Here we don't have diffusion and particles, but the activity can be considered as site occupancy, and we can map the threshold model with $K=2$ to an RD system with $n=2$ active neighbors necessary for creation and $m=1$ for spontaneous removal. In the presence of inhibitions $n>2$ is needed for creation at nodes with negatively weighted incoming links, which increases the inactive phase.

In the mean-field approximation the probability of site activity is $\rho$, and two active neighboring sites can occur in a $(N-1)(N-2) / 2$ way; thus the creation rate in case of a global acceptance probability $\Lambda$ is

$$
\frac{1}{2}(N-1)(N-2) \Lambda \rho^{2}(1-\rho) .
$$

Let us call $\Lambda(N-1)(N-2) / 2=\lambda$. For a full graph of $N$ nodes we can set up the rate equation

$$
\frac{d \rho}{d t}=\lambda \rho^{2}(1-\rho)-v \rho
$$

which in the $N \rightarrow \infty$ limit provides $\lambda_{c}=0$, but for finite graphs $\lambda_{c}>0$. In the steady state we have

$$
\lambda \rho^{2}(1-\rho)-v \rho=0 .
$$

By imposing the condition

$$
v=1-\lambda,
$$

we obtain

$$
\lambda \rho(1-\rho)-(1-\lambda)=0,
$$

which can be solved as

$$
\rho=\frac{\lambda \pm \sqrt{\lambda^{2}-4 \lambda(1-\lambda)}}{2 \lambda} .
$$

The solution is real and positive if

$$
\lambda>\lambda_{c}=4 / 5,
$$

providing a threshold within a system of size $N$

$$
\Lambda_{c}=\frac{8}{5(N-1)(N-2)}
$$

and an order parameter for $\Lambda \rightarrow \Lambda_{c}^{+}$

$$
\rho_{c}=1 / 2 \text {. }
$$

It is important to realize that in the $N \rightarrow \infty$ limit $\Lambda_{c} \rightarrow 0$, thus there is no inactive phase in the thermodynamic limit. But as real systems are always finite sized, we can observe this hybrid phase transition in them even in the mean-field limit. A similar phenomenon has recently been reported in contagion models [53]. Furthermore, in the case of the presence of inhibitory couplings the HPT at finite transition rate may survive the $N \rightarrow \infty$ limit in high dimensional systems.

At this transition point we can determine how the density approaches $\rho_{c}$ :

$$
\rho(t)-\rho_{c} \sim t^{-1}
$$

Thus here we find PL dynamical behavior even though the transition is discontinuous, as in other known hybrid or mixedorder transitions. For $\lambda<\lambda_{c}$ we have a $\rho=0$ stable solution and exponentially decaying activity. Right above the transition the steady-state density vanishes with a square-root singularity as in the case of k-core [8] or multiplex percolation hybrid transitions [10]:

$$
\left(\rho-\rho_{c}\right) \propto\left(\lambda-\lambda_{c}\right)^{1 / 2},
$$


but unlike the contact process [54] near multiple junctions [12], or the Kuramoto model with uniform frequency distribution [15], which thus belong to another hybrid universality class. In the following sections we investigate what happens to this HPT if we implement the threshold model on a hierarchical modular network (HMN).

\section{THRESHOLD MODEL ON HIERARCHICAL MODULAR NETWORKS}

In this section we describe the HMN models we use for the simulations. It is important to note that we believe that hierarchy is not relevant, but that modularity is what enhances RRs as in case of the study [49]. The models are motivated by brain networks originating from Kaiser and Hilgetag, who performed numerical studies to investigate the effects of different topologies on the activity spreading [55]. Their hierarchical model reflects general features of brain connectivity at large and mesoscopic scales, where the nodes were intended to represent cortical columns instead of individual neurons. The connections between them were modeled excitatory, since there appears to be no long-distance, inhibitory connections within the cerebral cortex [56].

The network was generated beginning with the highest level and adding modules to the next lower level with random connectivity within modules. Kaiser and Hilgetag explored hierarchical networks with different numbers of hierarchical levels and numbers of submodules at each level. The total, average node degree was set to a fixed value, motivated by comparative experimental studies. However, they investigated different topologies by varying the edge density across the levels. All the tested HMNs were small-world type, i.e., they exhibited infinite topological dimensions.

The spreading model they investigated was a two-state threshold model, in which nodes became activated with probability $\lambda$, when at least $K$ of their directly connected node neighbors were active at the same time or spontaneously deactivated, with probability $v$. Note that this model is very similar to RD models known in statistical physics [3,4], with a synchronous cellular automaton (SCA) update. Without loss of generality this algorithm produces faster dynamical scaling results for threshold models than those with random sequential updates.

In this paper we investigate versions of HMNs, which possess increasing edge density from top to bottom levels. Clearly, such topologies can be expected to be more suitable for activity localization and for RR effects.

One can also make a correspondence with the spatially embedded networks [57]. These networks have long links, with algebraically decaying probabilities in the Euclidean distance $R$ as

$$
p(R) \sim R^{-s} .
$$

We added random long links by level to level from top to bottom, similar to in Ref. [48]. The levels $l=0,1, \ldots, l_{\max }$ are numbered from bottom to top. The size of domains, i.e., the number of nodes in a level, grows as $N_{l}=4^{l+1}$ in the case of the four-module construction, related to a tiling of the $2 \mathrm{D}$

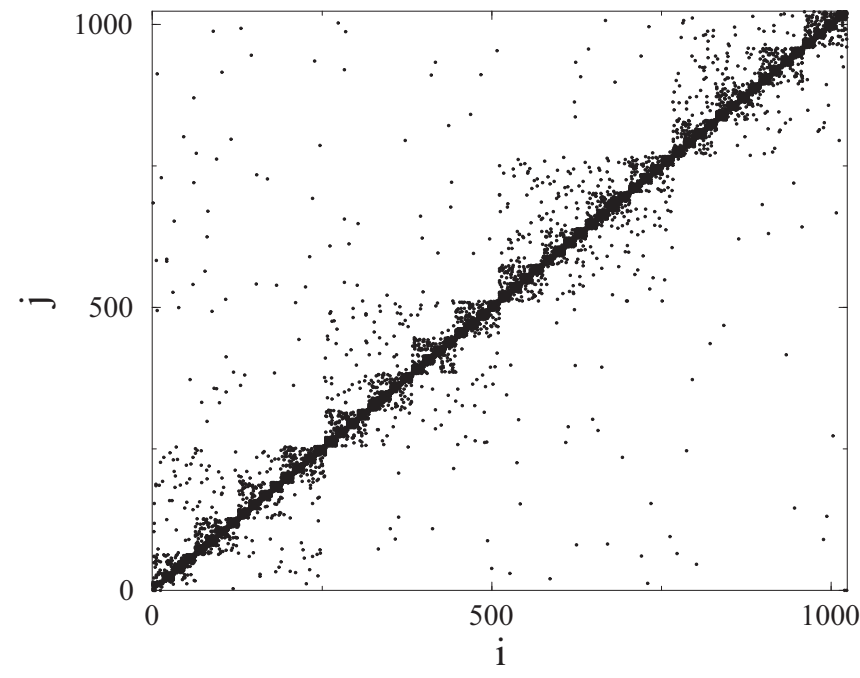

FIG. 1. Plot of the adjacency matrix of an $N=1024$-sized sample of the HMN2d graph. Black dots denote connections between nodes $i$ and $j$. The four-level structure is clearly visible in the blocks along the diagonal; additional long-range edges are scattered points around it.

base lattice, due to the rough distance level relation:

$$
p_{l}=b\left(\frac{1}{2^{s}}\right)^{l} .
$$

Here $b$ is related to the average degree $\langle k\rangle$ of nodes, which was prescribed to be $\langle k\rangle=12$ for this construction.

We connected nodes in a hierarchical modular way as if they were embedded in a regular two-dimensional lattice (HMN2d) as shown by the adjacency matrix in Fig. 1, similarly as in Ref. [48]. The four nodes of the level $l=0$ were fully connected. The single connectedness of the networks is guaranteed by additional linking of these four-node modules, by two edges to the subsequent ones: the first and the last nodes of module $(i)$ to the first node of module $(i+1)$. Accidental multiple connections were removed, and self-connections were not allowed. Note that the single connectedness at low level does not result in stable steady states in case of the threshold value $K=2$.

The in-degree distribution of four randomly selected graphs with $N=4096$ nodes can be seen in Fig. 2. The lowest in-degree is always $k_{i}^{\text {in }} \geqslant 5$. The modularity quotient of the networks is high: $Q>0.9$, defined by

$$
Q=\frac{1}{N\langle k\rangle} \sum_{i j}\left(A_{i j}-\frac{k_{i} k_{j}}{N\langle k\rangle}\right) \delta\left(g_{i}, g_{j}\right),
$$

where $A_{i j}$ is the adjacency matrix and $\delta(i, j)$ is the Kronecker delta function. The Watts-Strogatz clustering coefficient [58] of a network of $N$ nodes is

$$
C=\frac{1}{N} \sum_{i} 2 n_{i} / k_{i}\left(k_{i}-1\right),
$$

where $n_{i}$ denotes the number of direct edges interconnecting the $k_{i}$ nearest neighbors of node i. $C=0.295$ is about 10 times higher than that of a random network of the same size $C_{r}=0.0029$, defined by $C_{r}=\langle k\rangle / N$. The average shortest 


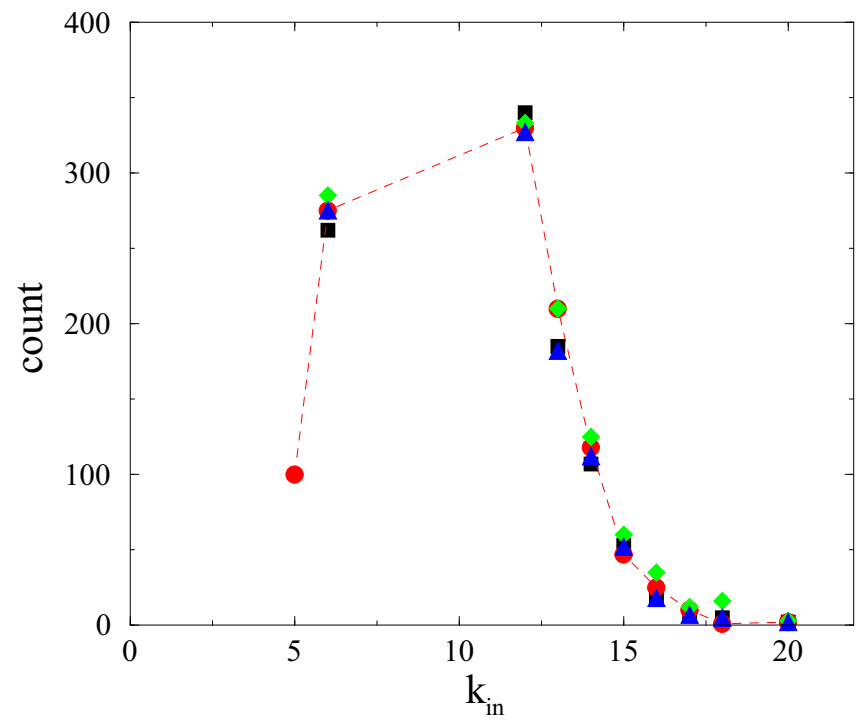

FIG. 2. In-degree distribution of four randomly selected $l=5$ HMN2d graphs.

path length is defined as

$$
L=\frac{1}{N(N-1)} \sum_{j \neq i} d(i, j),
$$

where $d(i, j)$ is the graph distance between vertices $i$ and $j$. In the case of this typical network $L=6.74$, about twice larger than that of the random network of same size: $L_{r}=3.615$, following from the following formula [59]:

$$
L_{r}=\frac{\ln (N)-0.5772}{\ln \langle k\rangle}+1 / 2 .
$$

So this is a small-world network, according to the definition of the coefficient [60]:

$$
\sigma=\frac{C / C_{r}}{L / L_{r}},
$$

because $\sigma=5.363$ is much larger than unity.

We estimated the effective topological dimension using the breadth-first search (BFS) algorithm: $d=4.18(5)$, defined by $N(r) \sim r^{d}$, where we counted the number of nodes $N(r)$ with chemical distance $r$ or less from the seeds and calculated averages over the trials. Note that this is just an estimate for the finite-sized graph, because we know that $d \rightarrow \infty$ is expected for $s=3$. It renders this model into the mean-field region, because for threshold models the upper-critical dimension is $d_{c} \leqslant 4$. Still, due to the heterogeneous structure, we find very nontrivial dynamical GP scaling behavior, as will be shown in the following sections.

\section{DYNAMICAL SIMULATIONS}

Time-dependent simulations were performed for single active seed initial conditions. It means that a pair of nodes is activated at neighboring sites: $x_{i}=x_{i+1}=1$, in an otherwise fully inactive system. It can trigger an avalanche, a standard technique in statistical physics to investigate critical initial slip [2]. We measured the spatiotemporal size $s=\sum_{i=1}^{N} \sum_{t=1}^{T} x_{i}$

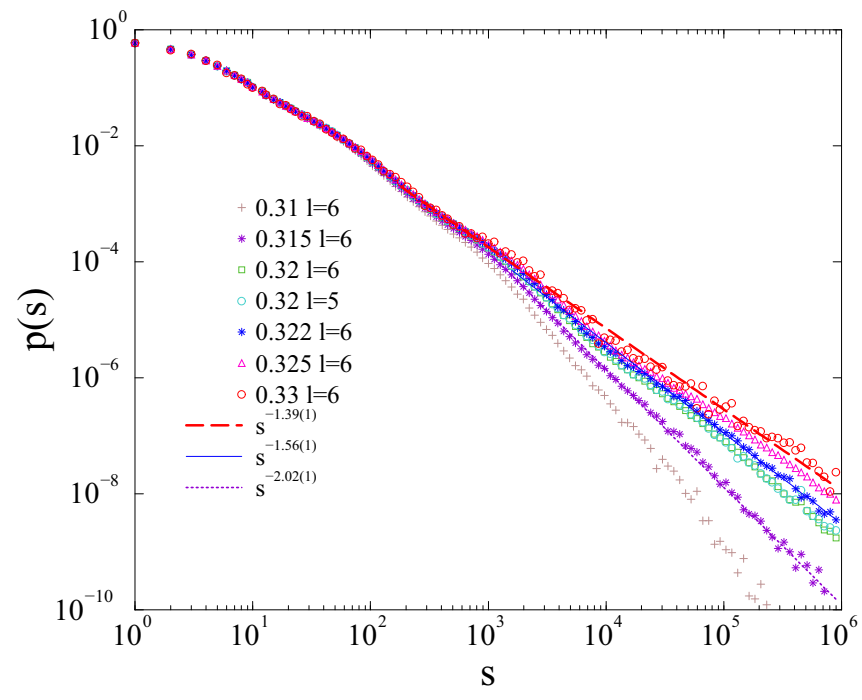

FIG. 3. Avalanche size distributions at different $\lambda$ branching rates, denoted by the symbols, in the presence of excitatory links in the HMN2d with $l=5,6$ levels. From top to bottom curves: $\lambda=0.33,0.325,0.322,0.32$ ( $l=5$ cyan and $l=6$ green $), 0.315$, 0.31. Dashed lines show PL fits for the tails: $s>1000$ at $\lambda=0.315$, $0.322,0.33$

and the duration of the avalanches $(T)$ for tens of thousands of random initial conditions: both initial sites and initial graph configurations. The graphs we investigated had $l=4,5,6,7$ levels, containing $N=1024$, 4096, 16384,65536 nodes, respectively. The average node degree was $\langle k\rangle \simeq 12$, after the low-level linking and the removal of accidental multiple edges. The ratio of short and long links was $\simeq 0.6$.

We have set $v=1-\lambda$ and updated the sites at discrete time steps, i.e., set the state variables $x^{\prime}(i)=1$ if it was inactive $x(i)=0$ and the sum of active neighbors $\sum_{j} x(j)$ exceeded $K=2$ with probability $\lambda$ or to $x^{\prime}(i)=0$ with probability $1-\lambda$ if it was active $x(i)=1$. Following a full sweep of sites we wrote $x(i)=x^{\prime}(i)$ for all nodes, corresponding to one Monte Carlo step (MC); throughout the study we measure time in MCs units. We have measured the density of active nodes $\rho(t)=1 / N \sum_{i=1}^{N} x_{i}$.

\section{A. Excitatory model}

The simulations were run for $T=10^{7} \mathrm{MCs}$, or until the system goes to a fully inactive state, corresponding to the end of the avalanche. We computed the probability density functions of avalanche sizes $p(s)$ and final survival time distributions $p(t)$. We repeated these simulations for different $\lambda$ branching rates by increasing its value. As Fig. 3 shows we don't see exponential decays as should be in the inactive phase of a mean-field model. Instead, there are PL-like tails for $\lambda>0.31$, modulated slightly by oscillations, which is a well-known phenomenon when discrete spatial periodicity is present, here the size of the modules. The slopes of the PL tails vary from $\tau=2.02$ to $\tau=1.39$ as we increase $\lambda$ from 0.315 to 0.33 .

Nonuniversal PL tails are more clearly visible on the avalanche survival time distributions plotted on the graph, 


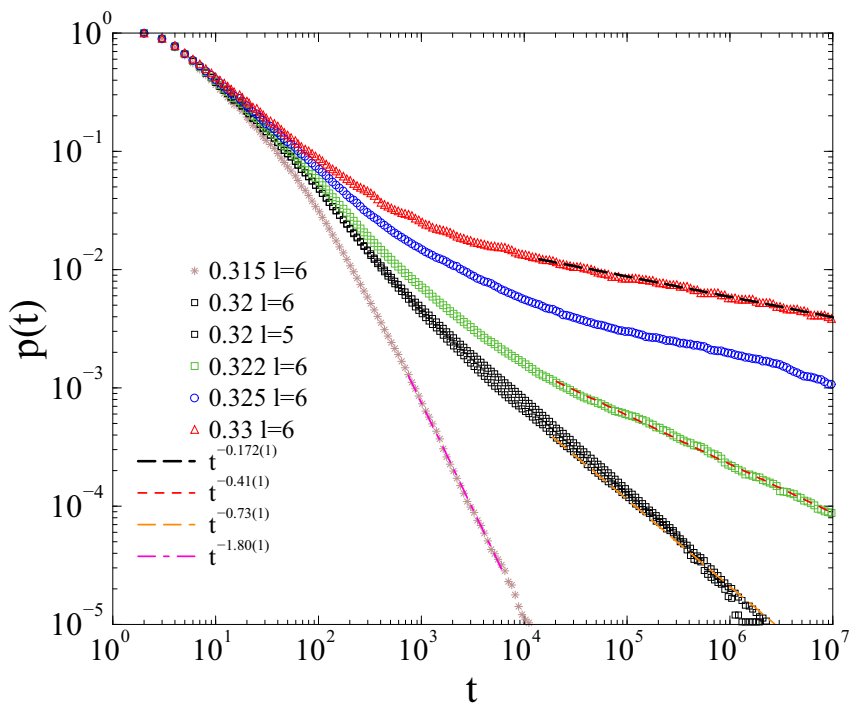

FIG. 4. Survival probability of the activity at different branching rates in the $K=2$ threshold model with excitatory links. From top to bottom curves: $\lambda=0.33,0.325,0.322,0.32(l=5$ and $l=6), 0.315$. Dashed lines show PL fits for the tails: $s>10^{4}$ at $\lambda=0.315,0.32$, $0.322,0.33$.

shown in Fig. 4. Here we can observe a greater variation moving from $\lambda=0.315$ with $\delta=1.80$ (1) to $\lambda=0.33$ with $\delta=0.172(1)$. The avalanche duration distributions can be deduced from these curves as the time integral, thus $\delta$ is related to the duration exponent of $P(t) \propto t^{-\tau_{t}}$ as

$$
\tau_{t}=1+\delta
$$

These nonuniversal PLs suggest that Griffiths effects are present, as reported in Ref. [48] for this model at different parameters. By repeating the simulations at different sizes: $l=5,6$ the distribution curves do not change within the error margin, and this size invariance implies the presence of real GPs.

The seminal experiments by Beggs and Plenz [31] reported neuronal avalanches with size $(s)$ dependence, defined as either the number of electrodes with suprathreshold activity or the sum of the potentials, according to a power law, $p(s) \propto$ $s^{-1.5}$. For the duration distribution of such events $P(t) \propto t^{-2}$, PL tails were observed. These exponents are in agreement with the mean-field (MF) exponents of the directed percolation (DP) criticality: $\tau=3 / 2, \tau_{t}=2$; see Ref. [3]. Mean-field exponents are expected to occur if the fluctuation effects are weak, when the system dimension is above the upper critical dimension $d_{c}$.

On the other hand, Palva et al. [61] have found that source-reconstructed $\mathrm{M} / \mathrm{EEG}$ data exhibit robust power-law, long-range time correlations and scale-invariant avalanches with a broad range of exponents: $1<\tau<1.6$ and $1.5<\tau_{t}<$ 2.4. These broad range exponent results have also been found in a recent cortical electrode experiment study on rodents [62]. An obvious explanation for this wide spread of critical exponents can be heterogeneity, which in the GP causes nonuniversal dynamical exponents [48,63-65].

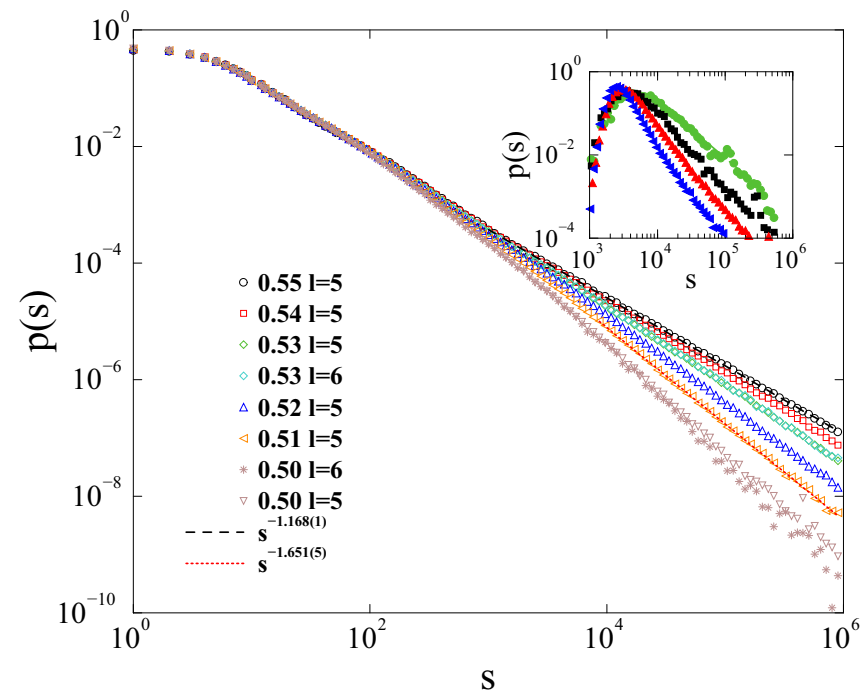

FIG. 5. Avalanche size distributions at different $\lambda$ branching rates, denoted by the symbols, in the presence of inhibitory links in HMN2d with $l=5,6$ levels. From top to bottom curves: $\lambda=0.55$, $0.54,0.53$ ( $l=5$ green and $l=6$ cyan $), 0.52,0.51,0.50(l=5$ triangle and $l=6$ diamond). Dashed lines show power-law fits for the tails of $\lambda=0.55,0.51$ cases, for $t>1000$. Inset: overlapping avalanches case for half-filled initial condition at $\lambda=$ $0.51,0.515,0.52,0.525$ (bottom to top symbols).

\section{B. Inhibitory model}

Although inhibitory links are not expected at long range links of the brain [56], we believe that our synthetic model may describe smaller cortical scales as well. As well, inhibitory mechanisms can occur in other phenomena with threshold dynamics. In the case of power grids, for example, feedback is applied to prevent catastrophic blackout avalanches, or in models of social/epidemic contagion, nodes with inhibitory properties may also exist. For simplicity we modeled the inhibitions by the introduction of links with negative weight contribution $\left(w_{i, j}\right)$ in the threshold comparison rule given by Eq. (1), although we think our results are easily transferred to the case of inhibitory nodes. As in Refs. [64,65], we randomly flipped the sign of $20 \%$ of the edges after the generation of the network.

The same analysis resulted in similar behavior as for the excitatory case. One can see $p(s)$ distributions with nonuniversal PLs ranging from $\lambda=0.5$ with $\tau=1.651$ (1) to $\lambda=0.55$ with $\tau=1.168(1)$ (Fig. 5). Finite-size dependence is not visible by changing the size from $N=4096$ to $N=16384$.

Usually it is believed that overlapping avalanches distort the scaling behavior. From the point of view of statistical physics, this would contradict universal asymptotic scaling behavior. Indeed we can see the same cumulative $p(s)$ distribution tails even in the case of starting the system from half-filled active state as shown in the inset of Fig. 5. The only difference is that the tails are shifted to larger $s$ values following an initial growth, which might not be observed in the case of short time measurements.

The $p(t)$ decays show GP behavior from $\lambda=0.505$ with $\delta=1.10(3)$ to $\lambda=0.52$ with $\delta=0.70(3)$ (Fig. 6). These values do not correspond to the ends of the GP; we did not aim 


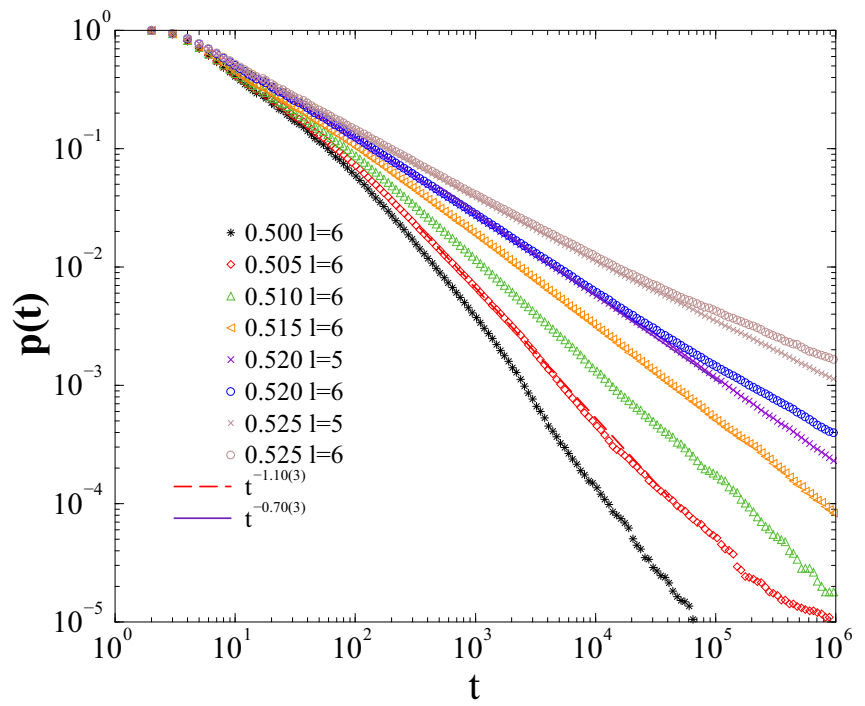

FIG. 6. Survival probability of the activity at different branching rates and $v=1-\lambda$ for the $K=2$ threshold model with levels: $l=$ 5,6 for the case with $20 \%$ of inhibitory links. From bottom to top symbols: $\lambda=0.5,0.505,0.510,0.515,0.520(l=5$ purple cross and $l=6$ blue circle), 0.525 ( $l=5$ brown cross and $l=6$ brown circle). Dashed lines are PL fits for the tails of $\lambda=0.505,0.52$ curves.

to determine them precisely as the exponents are nonuniversal. Furthermore, as we will show in Sec. V the determination of the upper limit of the GP, corresponding to the critical decay, is almost impossible with numerical simulations. Again the $\tau$ and the $\tau_{t}=1+\delta$ values lie within the range obtained by experiments.

\section{Inhibitory-refractory model}

Finally, we extended the inhibitory case study with the possibility of refractory states. Refractorieness means that, following an activation, nodes cannot fall back immediately to the inactive state on the next update; instead they stay for time $\Delta t$ in a refractory state. Thus they cannot be reactivated by the neighbors they excited. This refractoriness is generic in excitable systems and has been used in most of the neural studies $[18,66,67]$. One of the consequences of refractoriness is to induce oscillatory dynamics if $\Delta t$ is large enough and the spreading properties resemble annular growth, corresponding to dynamical isotropic percolation (DIP) [4]. However, real DIP occurs if reactivation is not possible, i.e., in the limit $\Delta t \rightarrow \infty$, and in a high dimension the avalanche scaling exponents of DIP are the same as those of DP [4,68]. Thus analytic studies or simulations in high dimensions do not show differences. In the extensive GP simulations we used $\Delta t=1$, but in the inset of Fig. 7 we show oscillatory activity behavior of a single run for $\Delta t=10, \lambda=0.8$, and $l=6$.

In Ref. [65] the GP behavior of the inhibitory-refractory threshold model was investigated on a large human connectome graph numerically. Nonuniversal $p(s)$ decays were reported with $1.4<\tau<1.91$. Here we can see this in the range $\lambda=0.39$ with $\tau=1.96(2)$ to $\lambda=0.43$ with $\tau=$ 1.39(1).

The avalanche survival probabilities (see Fig. 8) exhibit PL decay from $\lambda=0.40$ with $\delta=0.92$ (1) to $\lambda=0.43$ with

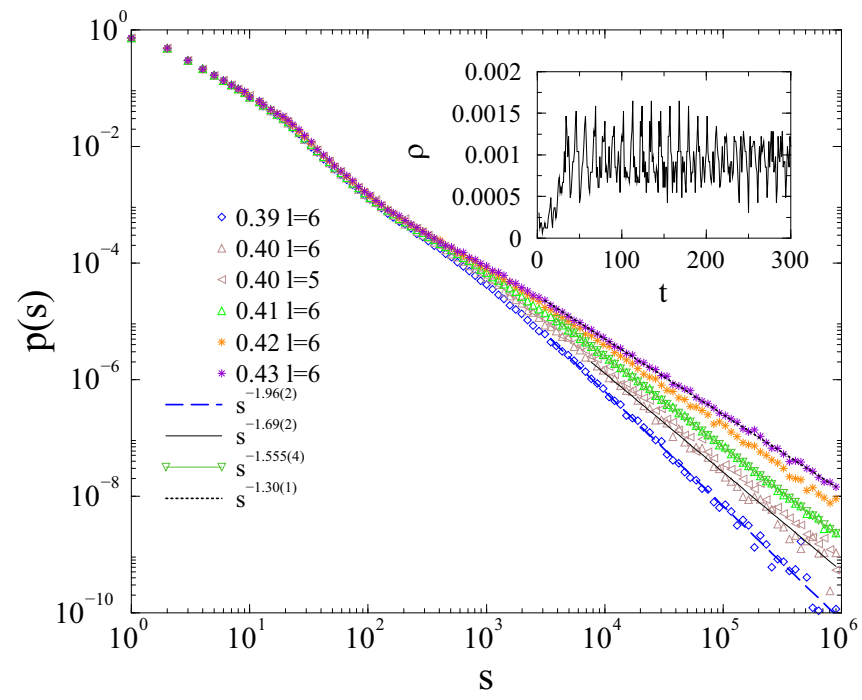

FIG. 7. Avalanche size distributions at different $\lambda$ branching rates, denoted by the symbols, in case of the refractory model, in the presence of inhibitory links in HMN2ds with $l=5,6$ levels. From bottom to top symbols: $\lambda=0.39,0.40(l=5$ left triangle and $l=6$ up triangle), $0.41,0.42,0.43$. Dashed lines are PL fits for the tails of $\lambda=0.39,0.4,0.41,0.43$ cases for $t>1000$. The inset shows the oscillatory behavior of $\rho(t)$ of a single run for $\Delta t=10$.

$\delta=0.39(1)$, so the duration exponent varies continuously: $1.39(1) \leqslant \tau_{t} \leqslant 1.92(1)$. Note that for similar models in Refs. [66,67] complex phase diagrams and nonuniversal PLs

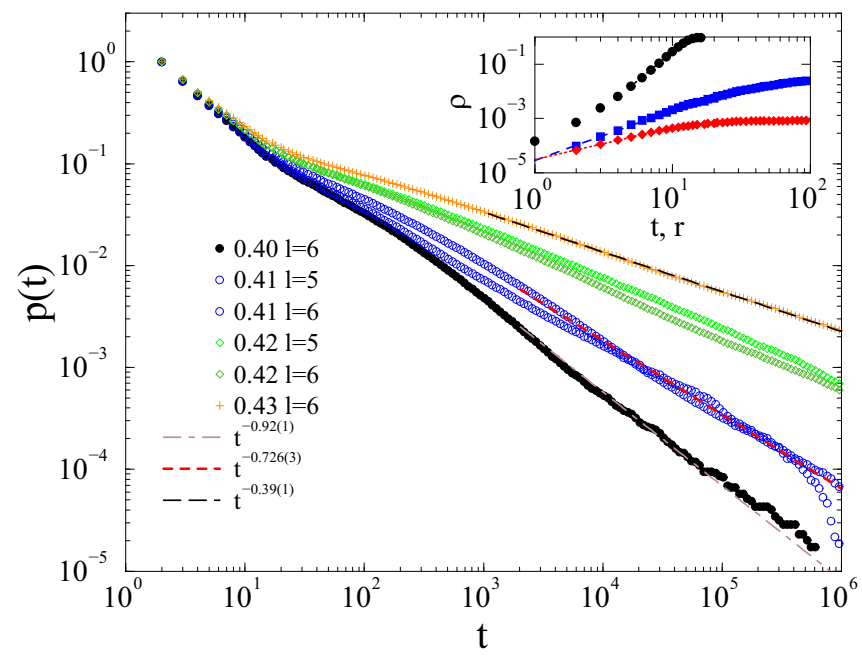

FIG. 8. Survival probability of the activity at different branching rates $\lambda$ for the levels $l=5,6$, in the case of the inhibitory-refractory model. From bottom to top symbols: $\lambda=0.40,0.41 \quad(l=5$ and $l=6), 0.42$ ( $l=5$ light green and $l=6$ dark green), 0.43 . Dashed lines show PL fits for $t>1000$ for the $\lambda=0.4,0.41,0.43$ cases. Inset: $\rho(t)$ at $\lambda=1, l=7$ averaged over $10^{5}$ realizations. Blue boxes: excitatory; red diamonds: inhibitory. Black bullets: BFS $\rho(r)$ results. Dashed lines are PL fits for the initial regions: $1 \leqslant t<$ 10) resulting in effective dimensions: $d_{\text {eff }}=1.84$ (3) (excitatory), $d_{\text {eff }}=1.19(1)$ (inhibitory), $d=4.18(5)$ (graph dimension estimated for $5<r<10$ ). 


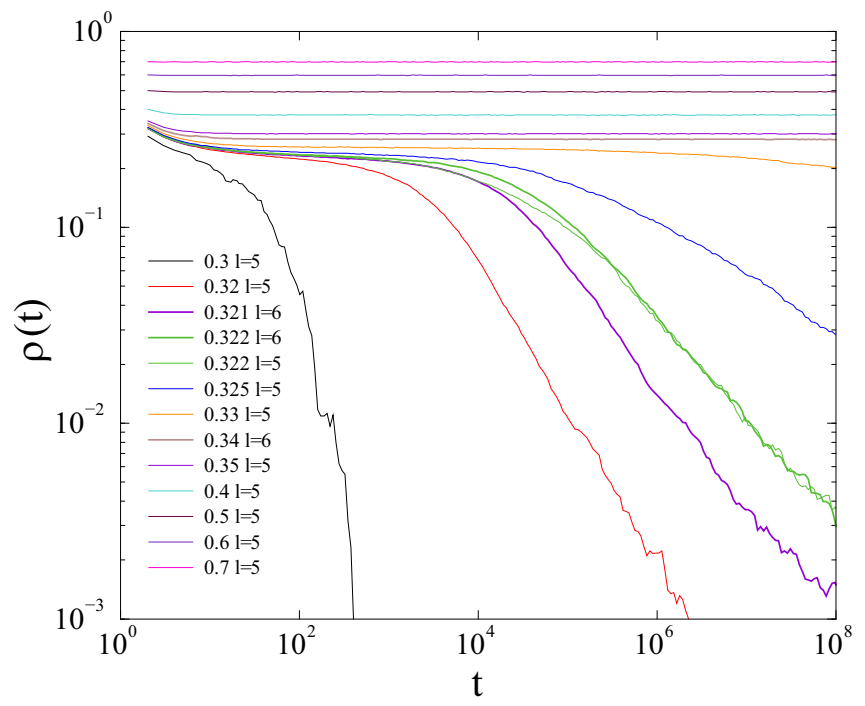

FIG. 9. Evolution of $\rho(t)$ for different $\lambda$ in case of starting from fully active state in the excitatory model with levels: $l=5,6$. From bottom to top symbols: $\lambda=0.30,0.32,0.321(l=6), 0.322,0.322$ $(l=6), 0.325,0.33,0.34(l=6), 0.35,0.4,0.5,0.6,0.7$.

have also been found and the possibility of GP has been pointed out.

\section{STEADY-STATE SIMULATIONS}

In order to determine the steady-state behavior we first performed long runs, up to $T=10^{8} \mathrm{MCs}$, by starting the system from fully active state or from randomly half-filled activity: $\rho(0)=0.5$. Figure 9 shows the results for the excitatory model. At $\lambda=0.3$ the activity density falls exponentially fast to zero. We can see nonuniversal PL tails for $0.32 \leqslant \lambda<$ 0.33 , in agreement with the seed simulations. At $\lambda=0.33$ the density does not saturate to a constant value. Examining it on log-lin scale and performing an average over thousands of independent samples it turns out that even the $\lambda=0.34$ curve decays very slowly. Only for $\lambda \geqslant 0.35$ can we can saturation, corresponding to an active steady state, thus, we estimate $\lambda_{c}=0.345(5)$. We plotted the steady-state saturation values in Fig. 10.

The same analysis has been done for the inhibitory and refractory-inhibitory cases, and one can observe the shift of $\lambda_{c}$ to higher values as the consequence of the model modifications. We show the results for the inhibitory network in Fig. 11. Again, slow activity decays were observed, ending up with visible PL tails for $0.51 \leqslant \lambda \leqslant 0.54$, while saturation starts from $\lambda_{c} \geqslant 0.80(1)$. The saturation value is $\rho_{c}=$ $0.685(1)$, so the discontinuity is large. In the region $0.54<$ $\lambda<0.80$ the curves do not saturate up to $T=10^{8} \mathrm{MCs}$, nor do they reach a scaling region. They belong to the inactive phase, but it is very difficult to distinguish them from other (logarithmic) decay forms.

We can see large jumps at the transition points in all cases, suggesting a discontinuous transition above the GP. It is very hard to locate the exact location of the transition points as stability disappears very slowly. This suggests that at the critical

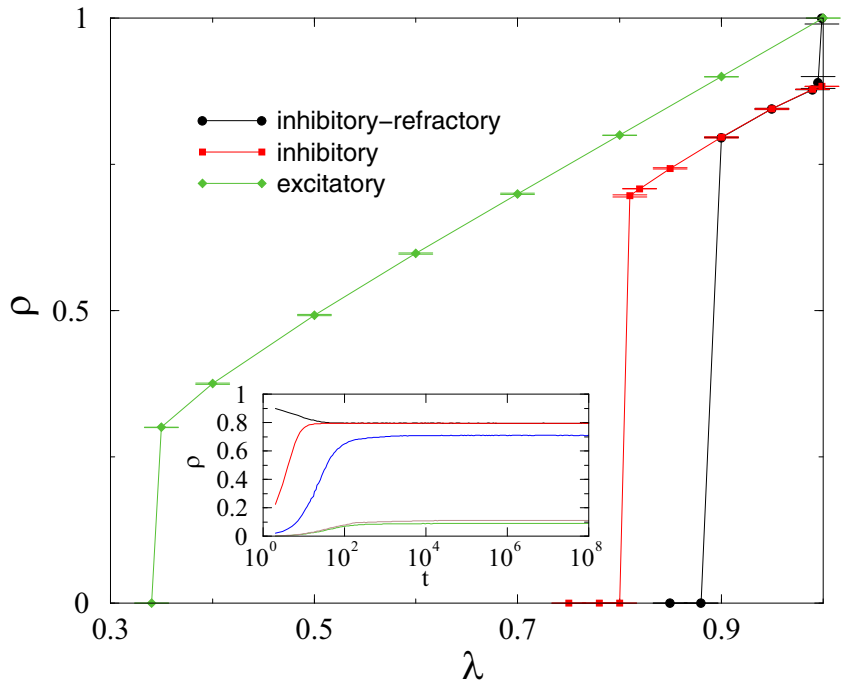

FIG. 10. Steady-state behavior for the excitatory, inhibitory, and refractory-inhibitory cases. Inset: evolution of $\rho$ in an inhibitory HMN2d with $N=4096$ for different initial activity densities: $\rho(0)=$ 0.0005, 0.001, 0.01, 0.1, 1 (bottom to top curves).

point logarithmic decay occurs like in case of the disordered DP [22].

We have also tried to start from other initial conditions than the full and single seed ones. As the inset of Fig. 10 shows we can see different saturation values for $\rho(0)<0.1$, which means we have multistability in case of low initial densities. This is the consequence of the fact that for low $\rho(0)$ only parts of the graph can be activated. Even though the networks are simply connected and the lowest in-degree is $k_{i}^{\text {in }}=5$, not

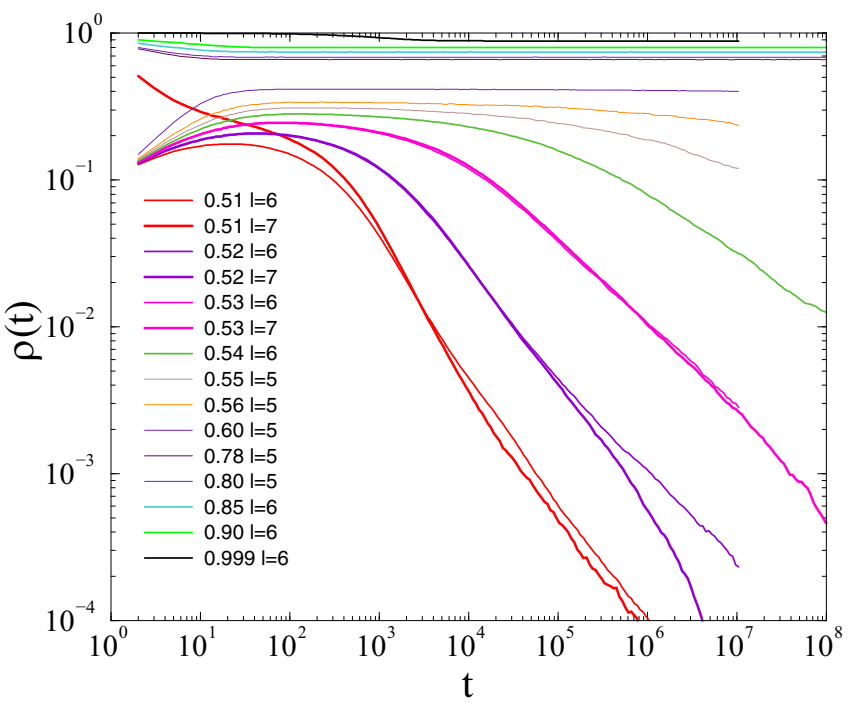

FIG. 11. Evolution of $\rho(t)$ for different $\lambda$, shown by legends in the case of starting from active states in the inhibitory model. Thick, normal, and thin lines correspond to $l=7, l=6$, and $l=5$, accordingly. From bottom to top curves: $\lambda=0.51,0.52,0.53,0.54$, $0.55,0.56,0.6,0.78,0.8,0.85,0.9,0.999$. The graph shows results with initial condition $\rho(0)=0.5$ for $\lambda \leqslant 0.6$, except for $\lambda=0.51$, $l=7$. In all the other cases $\rho(0)=1$ is applied. 
all nodes have two incoming links from the same neighbor, which is necessary for the activation. These nodes cannot be activated by a neighbor if they are on the "border" of an active territory, thus the graphs are practically fragmented from the activity point of view. This provides a mechanism for the emergence of GPs even in high dimensions, without breaking the conjecture provided in Ref. [47], according to which GPs and similar RR effects do not exist in networks with an infinite topological dimension.

Furthermore, we can see the emergence of discontinuous transition with multistable states, which can be considered bistable, for initial excitation with node fraction $\rho(0)>0.1$ converging to an "up" activity value, or by activation of single nodes, converging to a "down" value.

\section{CONCLUSIONS}

In conclusion we provided numerical evidence that strong heterogeneity effects in networks, coming from the modular structure, can result in a GP even if the topological dimension is high, where mean-field scaling would be expected. This is the consequence of fragmented activity propagation caused by the modular topology and the threshold. We can define effective dimensions of the these graphs by running seed simulations with $\lambda=1, v=0$ and measuring $\rho(t) \sim t^{d_{\text {eff }}}$. For this compact growth $\rho(t) \sim N(r)$, so $d_{\text {eff }}$ provides an estimate for the dimensionality, similarly to the BFS algorithm. This is reminiscent of similar methods, for instance, computing the spectral dimension of a network from random walk simulations [69-72]. While the topological dimension is a purely structural measure, $d_{\mathrm{eff}}$, as well as the spectral dimension are observables of processes operating on networks, providing insights into dynamical signatures of localization, slowing down and dynamical fragmentation. However, it has recently been shown that in models of HMNs the spectral dimension is not defined [73], thus our $d_{\text {eff }}$ can be a candidate to clarify relation of structure and slow dynamics. The inset of Fig. 8 shows that an initial scaling can be fitted for the excitatory case with $\rho(t) \sim t^{1.84(3)}$, while for the inhibitory case $\rho(t) \sim$ $t^{1.19(1)}$. Thus these effective, activity dimensions are less than $d_{c}$, much smaller than the topological dimension obtained by the BFS, which is also shown on the graph as the function of $r$.

Furthermore, the threshold-type models allow for the possibility to observe hybrid phase transitions, where order parameter discontinuity and multistability can coexist with dynamical scaling in a GP, thus they can model brain criticality as well as up/down states. External activation can then push the model among the multistable states if it is poised near the transition point.

The investigated $K=2$ discrete threshold model results can obviously be extended for higher $K$ values, and we expect to find similar behavior in continuous, integrate-and-fire type models on modular networks. Conversely, by duplicating the links we get back effectively the contact process [54] without RR effects and GPs. For neural systems our results imply that the functional and structural connectivity can be different. The effects of inhibition and refractive states have also been studied and emergence of oscillatory states has been shown. Our model results are applicable to a wide range of phenomena, like power grids, crack and fracture dynamics, and contagion.

\section{ACKNOWLEDGMENTS}

We thank Róbert Juhász for useful comments and discussions and Miguel Muñoz and Dante Chialvo for their valuable feedback. This work was supported by the National Research, Development and Innovation Office NKFIH under Grant No. K128989. We are grateful for access to the Hungarian National Supercomputer Network.
[1] J. Marro and R. Dickman, Nonequilibrium Phase Transitions in Lattice Models (Cambridge University Press, Cambridge, 2005).

[2] M. Henkel, H. Hinrichsen, and S. Lübeck, Non-equilibrium Phase Transition: Absorbing Phase Transitions (Springer Verlag, Amsterdam, 2008).

[3] G. Ódor, Rev. Mod. Phys. 76, 663 (2004).

[4] G. Ódor, Universality in Nonequilibrium Lattice Systems: Theoretical Foundations (World Scientific, Singapore, 2008).

[5] J. L. Cardy, J. Phys. A: Math. Gen. 14, 1407 (1981).

[6] H.-K. Janssen, M. Müller, and O. Stenull, Phys. Rev. E 70, 026114 (2004).

[7] W. Chan, F. Ghanbarnejad, and P. Grassberger, Nat. Phys. 11, 936 (2015).

[8] S. N. Dorogovtsev, A. V. Goltsev, and J. F. F. Mendes, Phys. Rev. Lett. 96, 040601 (2006).

[9] D. Lee, S. Choi, M. Stippinger, J. Kertész, and B. Kahng, Phys. Rev. E 93, 042109 (2016).

[10] G. J. Baxter, S. N. Dorogovtsev, J. F. F. Mendes, and D. Cellai, Phys. Rev. E 89, 042801 (2014).
[11] A. Bar and D. Mukamel, Phys. Rev. Lett. 112, 015701 (2014).

[12] R. Juhász and F. Iglói, Phys. Rev. E 95, 022109 (2017).

[13] P. Grassberger, Phys. Rev. E 95, 010102(R) (2017).

[14] R. Juhász and F. Iglói, Phys. Rev. E 97, 012111 (2018).

[15] D. Pazó, Phys. Rev. E 72, 046211 (2005).

[16] J. Gómez-Gardeñes, S. Gómez, A. Arenas, and Y. Moreno, Phys. Rev. Lett. 106, 128701 (2011).

[17] B. C. Coutinho, A. V. Goltsev, S. N. Dorogovtsev, and J. F. F. Mendes, Phys. Rev. E 87, 032106 (2013).

[18] A. Haimovici, E. Tagliazucchi, P. Balenzuela, and D. R. Chialvo, Phys. Rev. Lett. 110, 178101 (2013).

[19] S. Johnson, J. Marro, and J. J. Torres, PLoS ONE 8, e50276 (2013).

[20] P. Bak, C. Tang, and K. Wiesenfeld, Phys. Rev. Lett. 59, 381 (1987).

[21] D. R. Chialvo, Nat. Phys. 6, 744 (2010).

[22] T. Vojta, J. Phys. A: Math. Gen. 39, R143 (2006).

[23] R. B. Griffiths, Phys. Rev. Lett. 23, 17 (1969).

[24] G. Ódor, Phys. Rev. E 89, 042102 (2014). 
[25] M. Karsai, H.-H. Jo, and K. Kaski, Introduction, in Bursty Human Dynamics, SpringerBriefs in Complexity (Springer, Cham, 2018), pp. 1-6.

[26] W. Cota, S. C. Ferreira, and G. Ódor, Phys. Rev. E 93, 032322 (2016).

[27] Y. Imry and S.-K. Ma, Phys. Rev. Lett. 35, 1399 (1975).

[28] M. Aizenman and J. Wehr, Phys. Rev. Lett. 62, 2503 (1989).

[29] P. Villa Martín, J. A. Bonachela, and M. A. Muñoz, Phys. Rev. E 89, 012145 (2014).

[30] P. Villa Martín, M. Moretti, and M. A. Muñoz, J. Stat. Mech. (2015) P01003.

[31] J. Beggs and D. Plenz, J. Neurosci. 23, 11167 (2003).

[32] C. Tetzlaff, S. Okujeni, U. Egert, F. Wörgötter, and M. Butz, PLoS Comput. Biol. 6, e1001013 (2010).

[33] G. Hahn, T. Petermann, M. N. Havenith, S. Yu, W. Singer, D. Plenz, and D. Nikolić, J. Neurophysiol. 104, 3312 (2010).

[34] T. Ribeiro, M. Copelli, F. Caixeta, H. Belchior, D. Chialvo, M. Nicolelis, and S. Riberio, PLoS ONE 5, e14129 (2010).

[35] A. Ponce-Alvarez, A. Jouary, M. Privat, G. Deco, and G. Sumbre, Neuron 100, 1446 (2018).

[36] M. A. Muñoz, Rev. Mod. Phys. 90, 031001 (2018).

[37] D. Durstewitz, J. K. Seamans, and T. J. Sejnowski, Nat. Neurosci. 3, 1184 (2000).

[38] A. Kaltenbrunner, V. Gómez, and V. López, Neural Comput. 19, 3011 (2007).

[39] S. Scarpetta, I. Apicella, L. Minati, and A. de Candia, Phys. Rev. E 97, 062305 (2018).

[40] V. Buendía, P. Villegas, R. Burioni, and M. A. Muñoz, arXiv:2011.03263 (2020).

[41] O. Kinouchi and M. Copelli, Nat. Phys. 2, 348 (2006).

[42] I. Dobson, B. A. Carreras, V. E. Lynch, and D. E. Newman, Chaos 17, 026103 (2007).

[43] B. Schäffer, D. Witthaut, M. Timme, and V. Latora, Nat. Commun. 9, 1975 (2018).

[44] G. Ódor and B. Hartmann, Phys. Rev. E 98, 022305 (2018).

[45] M. J. Alava, P. K. V. V. Nukala, and S. Zapperi, Adv. Phys. 55, 349 (2006).

[46] S. Unicomb, G. Iniguez, and M. Karsai, Sci. Rep. 8, 3094 (2018).

[47] M. A. Muñoz, R. Juhász, C. Castellano, and G. Ódor, Phys. Rev. Lett. 105, 128701 (2010).

[48] G. Ódor, R. Dickman, and Gergely Ódor, Sci. Rep. 5, 14451 (2015).
[49] W. Cota, G. Ódor, and S. C. Ferreira, Sci. Rep. 8, 9144 (2018).

[50] M. Gastner and G. Ódor, Sci. Rep. 6, 27249 (2016).

[51] S. Di Santo, P. Villegas, R. Burioni, and M. Muñoz, Proc. Nat. Acad. Sci. USA 115, E1356 (2018).

[52] G. Ódor, Phys. Rev. E 67, 056114 (2003).

[53] W. Choi, D. Lee, J. Kertész, and B. Kahng, Phys. Rev. E 98, 012311 (2018).

[54] T. E. Harris, Ann. Probab. 2, 969 (1974).

[55] M. Kaiser, C. C. Hilgetag, and R. Kötter, Front. Neuroinform. 4, 112 (2010).

[56] P. E. Latham and S. Nirenberg, Neural Comput. 16, 1385 (2004).

[57] M. Barthelemy, C. R. Phys. 19, 205 (2018).

[58] D. J. Watts and S. H. Strogatz, Nature (London) 393, 440 (1998).

[59] A. Fronczak, P. Fronczak, and J. A. Hołyst, Phys. Rev. E 70, 056110 (2004).

[60] M. D. Humphries and K. Gurney, PLoS ONE 3, e0002051 (2008).

[61] J. Palva, A. Zhigalov, J. Hirvonen, O. Korhonen, K. LinkenkaerHansen, and S. Palva, Proc. Nat. Acad. Sci. USA 110, 3585 (2013).

[62] A. J. Fontenele, N. A. P. de Vasconcelos, T. Feliciano, L. A. A. Aguiar, C. Soares-Cunha, B. Coimbra, L. Dalla Porta, S. Ribeiro, A. J. A. Rodrigues, N. Sousa et al., Phys. Rev. Lett. 122, 208101 (2019).

[63] P. Moretti and M. A. Muñoz, Nat. Commun. 4, 2521 (2013).

[64] G. Ódor, Phys. Rev. E 94, 062411 (2016).

[65] G. Ódor, Phys. Rev. E 99, 012113 (2019).

[66] S. Moosavi, A. Montakhab, and A. Valizadeh, Sci. Rep. 7, 7107 (2017).

[67] M. Zarepour, J. I. Perotti, O. V. Billoni, D. R. Chialvo, and S. A. Cannas, Phys. Rev. E 100, 052138 (2019).

[68] M. A. Muñoz, R. Dickman, A. Vespignani, and S. Zapperi, Phys. Rev. E 59, 6175 (1999).

[69] R. Burioni and D. Cassi, Phys. Rev. Lett. 76, 1091 (1996).

[70] R. Burioni, D. Cassi, and A. Vezzani, Eur. Phys. J. B 15, 665 (2000).

[71] R. Burioni and D. Cassi, J. Phys. A: Math. Gen. 38, R45 (2005).

[72] A. P. Millán, J. J. Torres, and G. Bianconi, Phys. Rev. E 99, 022307 (2019).

[73] S. Esfandiary, A. Safari, J. Renner, P. Moretti, and M. A. Muñoz, Phys. Rev. Research 2, 043291 (2020). 